\title{
Changes in the levels of CAM kinase II and synapsin I caused by oxidative stress in the rat brain, and its prevention by vitamin $\mathrm{E}$
}

\author{
Nozomi Kaneai ${ }^{1}$, Koji Fukui ${ }^{2}$, Taisuke Koike ${ }^{3}$, Shiro Urano ${ }^{4 *}$ \\ ${ }^{1}$ Department of Applied Chemistry, Graduate School of Shibaura Institute of Technology, Tokyo, Japan \\ ${ }^{2}$ Division of Bioscience and Engineering, Shibaura Institute of Technology, Saitama, Japan \\ ${ }^{3}$ Eisai Food \& Chemical Co. Ltd., Tokyo, Japan \\ ${ }^{4}$ Life Support Technology Research Center, Shibaura Institute of Technology, Saitama, Japan \\ Email:m310502@shibaura-it.ac.jp,koji@sic.shibaura-it.ac.jp, t-koike@eisai-fc.co.jp, ${ }^{*}$ urano@shibaura-it.ac.jp
}

Received 20 September 2012; revised 30 October 2012; accepted 12 November 2012

\begin{abstract}
To define whether oxidative stress and aging induce abnormal dissociation of neurotransmitter-enclosing synaptic vesicles in rat brain nerve terminals, we assessed the activation of $\mathrm{Ca}^{+} /$calmodulin dependent protein kinase II (CAM kinase II) and changes in the levels of synapsin I, which is a synaptic vesicle-associated protein involved in the modulation of neurotransmitter release. Assessment of young rats subjected to hyperoxia-induced oxidative stress and normal aged rats revealed that synaptic CAM kinase II in the rat brain was markedly activated through oxidative stress and aging. In accordance with the activation of CAM kinase II, the levels of phosphorylated synapsin I increased significantly in nerve terminals. Furthermore, it was found that vitamin $E$ prevents these oxidative stress-induced abnormal processes in rat nerve terminals. These results suggest that oxidative stress and aging facilitate the mobilization of neurotransmitter-enclosing synaptic vesicles from the reserve pool in the nerve terminal, thereby inducing abnormal accumulation of synaptic vesicles in the synapse, and that vitamin $E$ inhibits this process in the brain through its antioxidative action.
\end{abstract}

Keywords: Oxidative Stress; CAM Kinase II; Synapsin I; Neurotransmission; Vitamin E

\section{INTRODUCTION}

Among the various organs, the brain is considered to be more susceptible to oxidative stress than other organs, leading to neuronal oxidative damage and neurodegenerative disorders such as Alzheimer's disease (AD) [1]. In fact, it has been confirmed that there are increased

\footnotetext{
${ }^{*}$ Corresponding author.
}

regional levels of oxidative stress in the $\mathrm{AD}$ brain, and hence oxidative stress is known to be involved in several neurodegenerative disorders characterized by progressive cognitive deficits $[2,3]$. In accordance to the oxidative stress theory of neurodegeneration, our previous findings revealed that normal aged rats and young rats subjected to oxidative stress exhibit several kinds of neuronal damage in the brain; specifically, swollen mitochondria, pigmentation, deformed nuclei, and the abnormal accumulation of neurotransmitter-enclosing synaptic vesicles in nerve terminals. Furthermore, it was found that the release of acetylcholine from nerve terminals is markedly decreased $[4,5]$. In accordance with these phenomena, normal aged rats and young rats subjected to oxidative stress showed marked cognitive impairment [6]. These observations suggest that oxidative stress induces damage in the nervous system, resulting in a decline in neurotransmission. This is supported by the fact that neurotransmitter-enclosing synaptic vesicles accumulate to abnormal levels in nerve terminals as a result of oxidative stress [4], and that the fusion of synaptic vesicles with pre-synaptic plasma membranes during neurotransmission is decreased. Decreased synaptic fusion is thought to be due to either the loss of docking proteins (soluble $\mathrm{N}$-ethylmaleimide-sensitive fusion protein attachment protein receptor, SNARE proteins) prior to the fusion process [7] or by damage to the lipid-mixing process caused by lipid peroxidation of both membranes [8].

It has been proposed that neurotransmission is modulated by the synapsin proteins in pre-synaptic terminals $[9,10]$. Synapsins are abundant neuronal substrates for cAMP- and $\mathrm{Ca}^{+} /$calmodulin dependent protein kinases. Synapsin I binds with high affinity to synaptic vesicles as well as cytoskeletal actin filaments [9]. Binding of synapsin I to synaptic vesicles enables joining of vesicles to each other and/or to pre-synaptic actin filaments, thereby maintaining a reserve pool of vesicles at the nerve ter- 
minal $[9,10]$. In response to neuronal signals, synapsin I is phosphorylated by CAM kinase II, which reduces its affinity to synaptic vesicles and actin filaments; thereby mobilizing the vesicles to pre-synaptic membranes for neurotransmission [9].

Since oxidative stress and aging induce neurodegeneration in the rat brain [5], it is reasonable to infer that regulation of neurotransmission by synapsin $\mathrm{I}$ is decreased by these processes. Although the pre-synaptic roles of synapsin I and CAM kinase II have been intensively studied, changes in CAM kinase II activation and synapsin I levels caused by oxidative stress and aging are not well-understood. The present study aims to determine whether or not oxidative stress increases CAM kinase II activity and decreases synapsin I levels, leading to abnormal neurotransmission.

Furthermore, since it is generally recognized that vitamin $\mathrm{E}$ acts as a physiologically potential antioxidant for scavenging reactive oxygen species (ROS) generated through oxidative stress in living tissue, and that vitamin E can prevent and/or inhibit oxidative damage in several neurodegenerative diseases such as vascular dementia and Alzheimer's disease, resulting in the maintenance of homeostasis in function [11], we assessed whether or not vitamin $\mathrm{E}$ can protect against changes in synapsin I levels caused by oxidative stress.

\section{MATERIALS AND METHODS}

\subsection{Materials}

Animals: All animal experiments were performed with the permission of the Animal Protection and Ethics Committee of the Shibaura Institute of Technology. 4-wk-old and aged (25-mo-old) male Wistar rats were fed ad libitum with a standard diet ( $\alpha$-tocopherol content: $3-5 \mathrm{mg}$ / $100 \mathrm{~g}$ diet $)$, or a vitamin E-supplemented diet $(250 \mathrm{mg} /$ $100 \mathrm{~g}$ diet). Young rats were fed for $9 \mathrm{wks}$, from $4 \mathrm{wks}$ of age, followed by exposure to oxidative stress $(100 \%$ oxygen) at $20^{\circ} \mathrm{C}$ for $48 \mathrm{~h}$ in an oxygen chamber. Aged rats were fed the vitamin E-supplemented diet for $9 \mathrm{wks}$ from 23-mo of age. Vitamin E-deficient rats were fed a vitamin E-deficient diet (Funakoshi Nojyo, Chiba, Japan) for 9 wks from 4 wks of age; no tocopherols were detected by HPLC.

Chemicals: Ficoll, dithiothreitol and leupeptin were purchased from Sigma Chemical Co. (St. Louis, MO, USA); PIPES (Piperazine-1,4-bis (2-ethnesulfonic acid) and CHAPS (3-[(3-Cholamidopropyl)dimethyl-ammonio] propane-sulfonic acid) from Dojindo Laboratories $(\mathrm{Ku}-$ mamoto, Japan); pepstatin-A and PMSF (Phenylmethylsulfonyl fluoride) from Wako Pure Chemical Co. Ltd., (Osaka, Japan); aprotinin from Takara Bio Inc. (Ohtsu, Japan); Can Get Signal solution 1 and 2 from TOYOBO Co. Ltd., (Osaka, Japan); ECL Prime Western Blotting
Detection Reagent from GE Healthcare Japan Co. Ltd., (Tokyo, Japan); and $\beta$-galactodidase from Calbiochem (San Diego, CA, USA). All other chemicals were of the highest grade available.

Antibodies: The CaMK II- $\alpha$ antibody was purchased from Cell Signaling Technology, Inc. (Danvers, MA, USA); anti tau phospho specific [Ser416] polyclonal antibody (anti-CaMK II) was from Trans Genic Inc. (Kumamoto, Japan); synapsin monoclonal antibody (223) and rabbit anti-phospho synapsin-I (Ser 9) polyclonal antibody were from Enzo Life Sciences Inc. (Farmingdale, NY, USA). The IgG fraction of a polyclonal rabbit antiserum to $\beta$-galactosidase was obtained from Nordic Immunological Laboratories, Inc. (Tilburg, Netherlands). Horseradish peroxidase (HRP)-conjugated goat IgG fraction against mouse IgG and HRP-conjugated donkey anti-rabbit IgG were purchased from Promega Corporation (Madison, WI, USA).

\subsection{Isolation of Synaptosomes}

Synaptosomes were isolated from whole brain according to the procedure of Dodd et al. [12], with minor modifications. After rats were decapitated, the brains were immediately removed and homogenized in ice-cold $10 \mathrm{mM}$ Tris-HCl buffer ( $\mathrm{pH}$ 7.4) containing $0.32 \mathrm{M}$ sucrose and $1 \mathrm{mM}$ EDTA with a glass/Teflon homogenizer using 12 strokes with a tight-fitting pestle. The homogenate was centrifuged at $750 \times \mathrm{g}$ at $4^{\circ} \mathrm{C}$ for $3 \mathrm{~min}$. The resulting pellet was re-homogenized and centrifuged as described above. The two supernatant fractions were combined and centrifuged at $20,000 \times \mathrm{g}$ at $4^{\circ} \mathrm{C}$ for $10 \mathrm{~min}$. The pellet was re-suspended in $2 \mathrm{~mL}$ of a $12 \%$ Ficoll solution in Tris-HCl buffer ( $\mathrm{pH}$ 7.4) and re-homogenized with 5 strokes of a loose-fitting pestle as described above. The homogenate was centrifuged at $150,000 \times \mathrm{g}$ at $4^{\circ} \mathrm{C}$ for 1 h on 3-step discontinuous Ficoll density gradients consisting of $12 \%, 7.5 \%$ and $0 \%$ Ficoll. The band at the interface between the $12 \%$ and $7.5 \%$ Ficoll density layers was collected and washed with phosphate buffered saline (PBS), and centrifuged at $5800 \times \mathrm{g}$ at $4^{\circ} \mathrm{C}$ for $15 \mathrm{~min}$. The band was suspended in PBS and used as the synaptosomal fraction for further experimentation. An assessment of synaptosomal purity in previous reports indicated that this fraction consists of almost pure synaptosomes [11].

\subsection{Immunoblotting Analysis of Synapsin-I and CAM Kinase II}

An aliquot of the synaptosomal suspension (protein content: $5 \mu \mathrm{g}$ for analyses of CAM kinase II and CAM kinase II- $\alpha, 4 \mu \mathrm{g}$ for synapsin-I, and $18 \mu \mathrm{g}$ for phospho synapsin-I) was homogenized three times in an ice bath for 5 seconds using an ultrasonic homogenizer. The ho- 
mogenate was dissolved into $5 \mu \mathrm{L}$ of a CHAPS cell extract buffer (100 mM PIPES, $4 \mathrm{mM}$ EDTA, 0.2\% (w/v) CHAPS, $110 \mathrm{mM}$ DTT, $40 \mu \mathrm{g} / \mathrm{mL}$ leupeptin, $20 \mu \mathrm{g} / \mathrm{mL}$ pepstatin-A, $20 \mu \mathrm{g} / \mathrm{mL}$ aprotinin, $2 \mathrm{mM}$ PMSF, pH 6.8) and mixed with a solution of $\beta$-galactosidase $(1 \mu \mathrm{L}, 500$ $\mu \mathrm{g} / \mathrm{mL}$ in PBS). After the mixture was incubated for 20 min in an ice bath, $125 \mathrm{mM}$ Tris- $\mathrm{HCl}$ buffer $(\mathrm{pH}$ 6.8), containing 4\% sodium dodecyl sulphate (SDS), 20\% glycerol, $0.01 \%(\mathrm{w} / \mathrm{v})$ bromophenol blue and $0.55 \%(\mathrm{w} / \mathrm{v})$ mercaptoethanol, was added. The mixture was fractionated using 10\% SDS-polyacrylamide gel electrophoresis (PAGE) together with a protein molecular weight marker (NIPPON Genetics Co. Ltd., Tokyo, Japan). Separated proteins were transferred onto Immobilon transfer membranes (Millipore, Bedford, MA, USA). The membranes were washed twice with a $25 \mathrm{mM}$ Tris buffer, containing $137 \mathrm{mM} \mathrm{NaCl}, 2.7 \mathrm{mM} \mathrm{KCl}$, and $0.05 \%$ Tween-20 (TBS-T, $\mathrm{pH}$ 7.4). After blocking non-specific binding sites for $1 \mathrm{~h}$ with a blocking solution (TBS-T with $2 \%$ bovine serum albumin (BSA), $\mathrm{pH} 7.4$ ), blots were incubated with anti-CaMK II- $\alpha$ (1:1000), anti-CaMK II (1:1000), anti-synapsin I (1:500), anti-phospho synapsinI $(1: 1000)$ and anti- $\beta$-galactosidase antibodies $(1: 1000)$ in Can Get Signal solution 1 at $4^{\circ} \mathrm{C}$ for $12 \mathrm{~h}$. Blots were washed with TBS-T, and incubated with horseradish (HRP)-linked anti-mouse secondary antibody (1:2000) in Can Get Signal solution 2 for synapsin-I antibody, or an anti-rabbit antibody for all other antibodies. The HRPlabeled antibodies were detected with the enhanced chemiluminescence detection system using a luminoana-

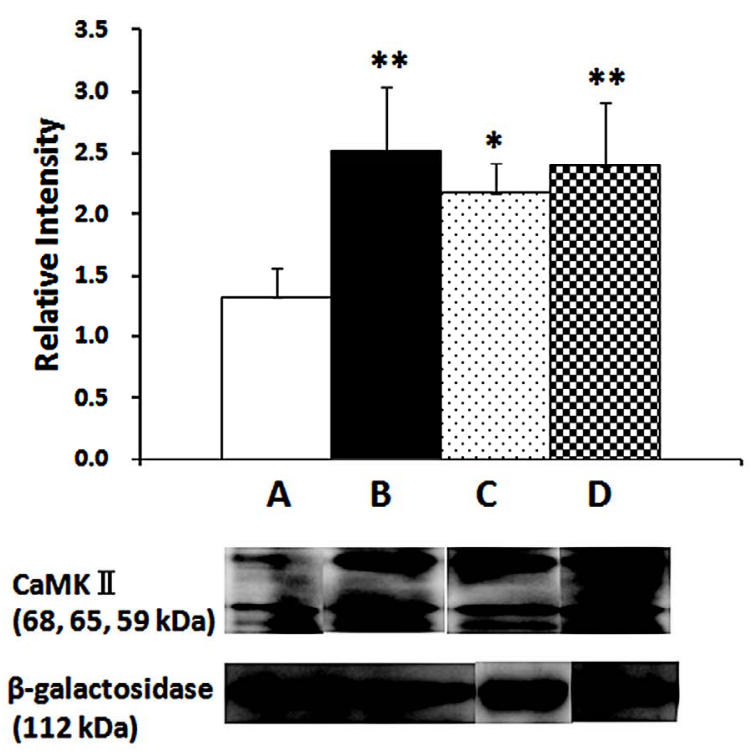

(a) CaMK II lyzer (Las-3000, FUJI film imaging Co. Ltd., Tokyo, Japan). $\beta$-Galactosidase was used as an internal standard.

\subsection{Statistical Analysis}

Results are presented as means \pm SE. All data were analyzed with a Student's t-test or a one-way ANOVA followed by a Dunnett's t-test. The experimental data were considered to be statistically different when $\mathrm{p}$-values were less than 0.05 .

\section{RESULTS}

\subsection{CAM Kinase II Activation in the Brain through Oxidative Stress and Aging}

As shown in Figures 1(a) and (b), young rats subjected to oxidative stress showed a marked increase in the expression of CAM kinase II in brain nerve terminals. In young animals, oxidative stress increased total CAM kinase II and CAM kinase II- $\alpha$ level versus normal controls, by $89.5 \%$ and $107.9 \%$, respectively. A similar tendency was observed in normal aged rat nerve terminals (63.6\% and 86.5\%, respectively) and vitamin E-deficient young rats maintained under normal conditions $(79.0 \%$ and $82.3 \%$, respectively). Since the effects of aging are thought to be due to an accumulation of oxidative damage in living tissues through oxidative stress experienced over long periods of time [13,14], and that vitamin Edeficient young rats experience oxidative stress under normal conditions, normal aged rats and vitamin E-deficient young rats showed similar increases in brain CAM
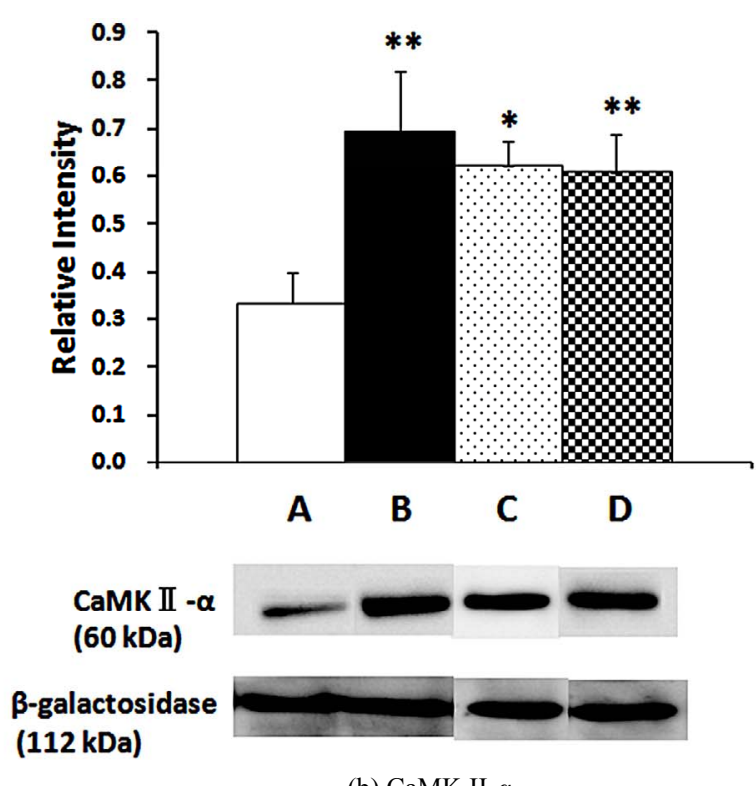

(b) CaMK II- $\alpha$

Figure 1. Changes in the expression of CAM kinase II (a), and CAM kinase II- $\alpha$ (b) caused by oxidative stress. A, control rats; B, young rats subjected to oxidative stress; $\mathrm{C}$, normal aged rats; D, vitamin E-deficient young rats. (a) ${ }^{*} \mathrm{P}<0.01$ versus control, ${ }^{* *} \mathrm{P}<$ 0.03 versus control; (b) ${ }^{*} \mathrm{P}<0.03$ versus control, ${ }^{* *} \mathrm{P}<0.03$ versus control; $\mathrm{n}=5$ for each group. Intensity values are presented as relative to the internal standard $\beta$-galactosidase and were determined using a luminoanalyzer. 
kinase II expression and, presumably, its activity. The CAM kinase II antibody, which is partly cross-reactive to the serine 416 residue of tau proteins, was used to assess total levels of the CAM kinase II family, resulting in three bands (59, 65 and $68 \mathrm{KD})$ being observed on the blots (Figure 1(a)). To assess the expression of the CAM kinase II subtype that is responsible for phosphorylation of synapsin I, the CAM kinase II- $\alpha$ antibody was used. As expected, the relative intensity of CAM kinase II- $\alpha$ was found to be lower than for total CAM kinase II. However, it is evident that oxidative stress induces abnormal activation of the CAM kinase II- $\alpha$ enzyme in brain nerve terminals.

\subsection{Phosphorylation of Brain Synapsin I through Oxidative Stress and Aging}

To determine whether synapsin I is phosphorylated in brain nerve terminals in a manner consistent with the activation of CAM kinase II, we assessed changes in the expression of synapsin I caused by oxidative stress and aging using immunoblotting. It was found that brain synapsin I levels were marked decreased in young rats subjected to oxidative stress, normal aged rats and normoxic vitamin E-deficient rats (Figure 2(a)). In contrast, the expression of phosphorylated synapsin I was markedly increased in all three groups, compared to normal young control rats (Figure 2(b)).
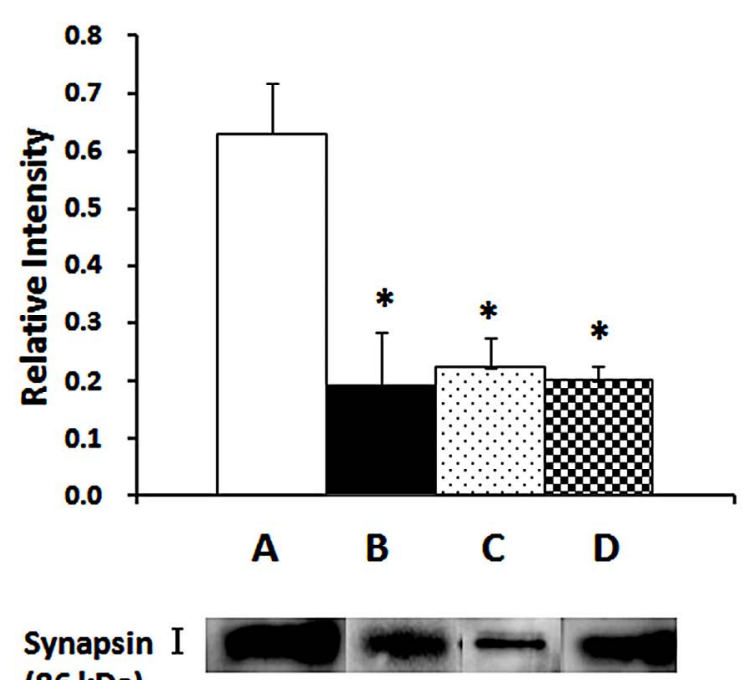
(86 kDa)

\section{$\beta$-galactosidase (112 kDa)}

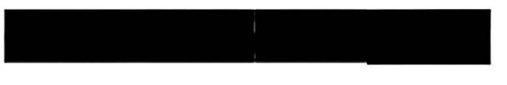

(a) Synapsin I

\subsection{Inhibition of CAM Kinase II Activation and Phosphorylation of Synapsin I by Vitamin E Treatment}

Based on the results of this study, vitamin E could potentially inhibit the abnormal events in the neurotransmission process cased by oxidative stress. Although young rats and normal aged rats fed a vitamin E-supplemented diet showed a tendency to inhibit the oxidative stressinduced increase in the expression of total CAM kinase II (Figure 3(a)), no significant differences were detected. However, the expression of oxidative stress-induced CAM kinase II- $\alpha$ in rats fed vitamin E-supplemented diet was markedly decreased (46.8\%, Figure 3(b)).

Furthermore, vitamin E inhibited oxidative stress-induced decreases in synapsin I levels and increases in phosphorylated synapsin I (185.4\% and 55.4\%, respectively; Figures 4(a) and (b)). A similar tendency was observed in normal aged rats fed a vitamin E-supplemented diet. Vitamin E inhibited the decrease in the levels of synapsin I (56.5\%) and the increase in phosphorylated synapsin I (23.8\%).

\section{DISCUSSION}

It is well recognized that oxidative stress occurs in living tissues during periods of imbalance between ROS generation and detoxification by antioxidants. ROS that overcome the antioxidative barrier induce oxidative damage
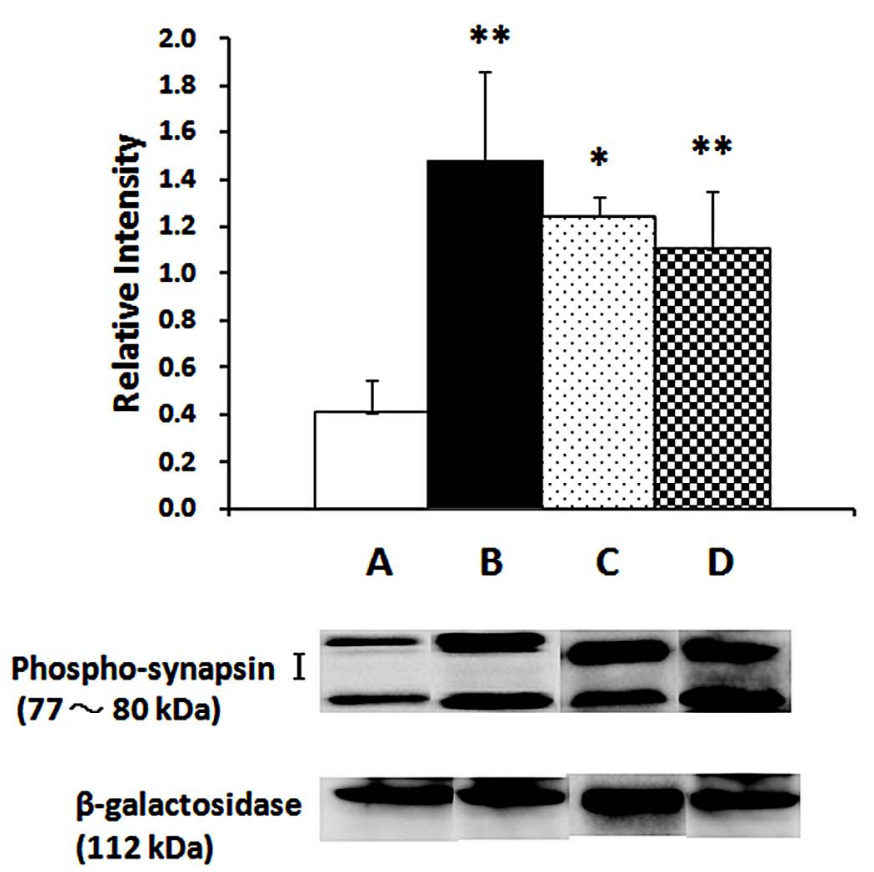

(b) Phosphrylated synapsin I

Figure 2. Changes in the expression of synapsin I (a), and phosphorylated synapsin I (b) caused by oxidative stress. A, control rats; B, young rats subjected to oxidative stress; $\mathrm{C}$, normal aged rats; D, vitamin E-deficient young rats. (a) ${ }^{*} \mathrm{P}<0.01$ versus control; (b) ${ }^{*} \mathrm{P}<$ 0.03 versus control, ${ }^{* *} \mathrm{P}<0.03$ versus control; $\mathrm{n}=5$ for each group. Intensity values are presented as relative to the internal standard $\beta$-galactosidase and were determined using a luminoanalyzer. 


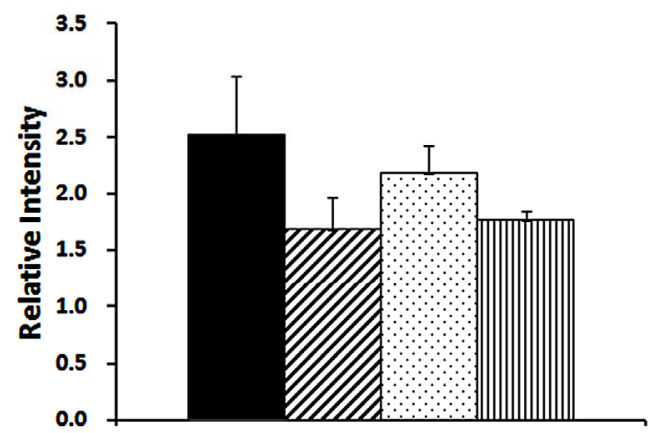

A $\quad$ B $\quad$ C $\quad$ D

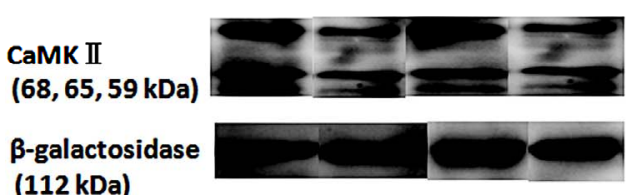

(a) CaMK II

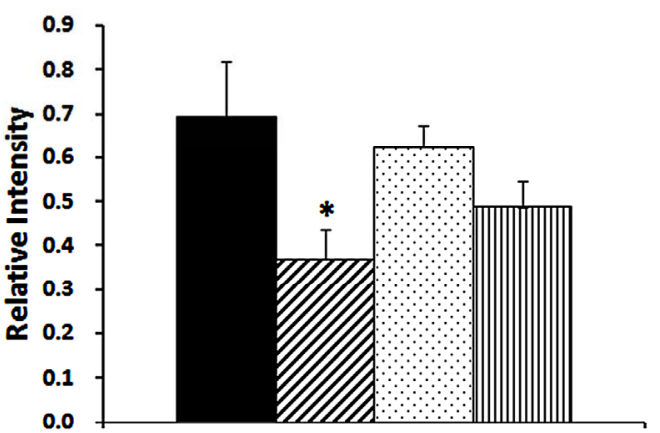

A B C D

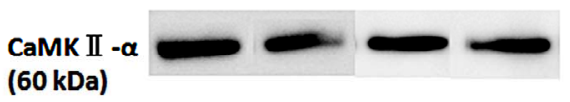

$\beta$-galactosidase (112 kDa)

(b) CaMK II- $\alpha$

Figure 3. Inhibitory effect of vitamin E on oxidative stress-induced expression of CAM kinase II (a), and CAM kinase II- $\alpha$ (b). A, young rats subjected to oxidative stress; $\mathrm{B}$, young rats fed a vitamin E-supplemented diet and subjected to oxidative stress; $\mathrm{C}$, normal aged rats; $\mathrm{D}$, aged rats fed a vitamin E-supplemented diet. ${ }^{*} \mathrm{P}<0.03$ versus young rats subjected to oxidative stress; $\mathrm{n}=5$. Intensity values are presented as relative to the internal standard $\beta$-galactosidase and were determined using a luminoanalyzer.

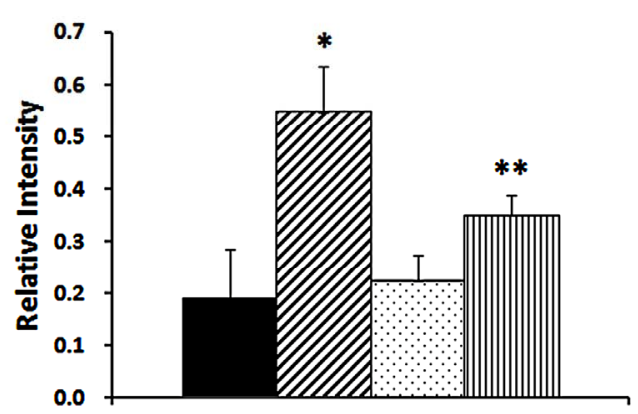

A $B$ C

Synapsin I (86 kDa)

\section{B-galactosidase} (112 kDa)

(a) Synapsin I

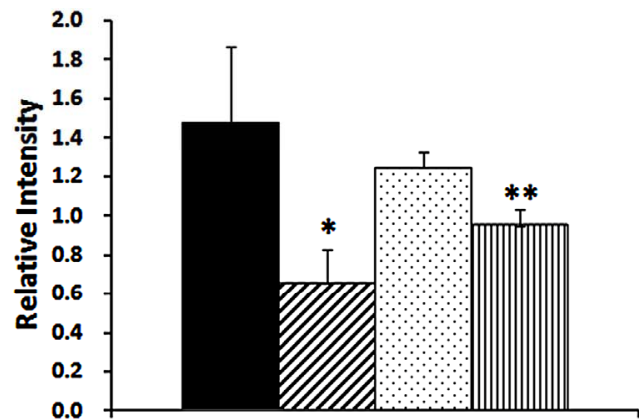

A B C D

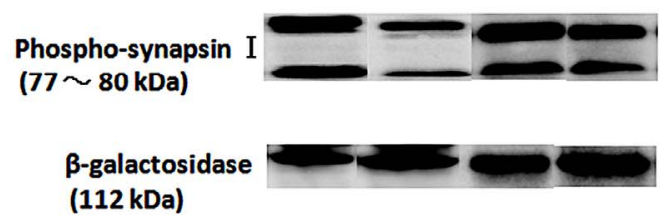

(b) Phosphrylated synapsin I

Figure 4. Inhibitory effect of vitamin E on oxidative stress-induced expression of synapsin I (a), and phosphorylated synapsin I (b). A, young rats subjected to oxidative stress; B, young rats fed a vitamin E-supplemented diet and subjected to oxidative stress; C, normal aged rats; $\mathrm{D}$, aged rats fed a vitamin E-supplemented diet. (a) ${ }^{*} \mathrm{P}<0.03$ versus young rats subjected to oxidative stress, ${ }^{* *} \mathrm{P}<$ 0.05 versus normal aged rats; (b) ${ }^{*} \mathrm{P}<0.05$ versus young rats subjected to oxidative stress, ${ }^{* *} \mathrm{P}<0.02$ versus normal aged rats; $\mathrm{n}=5$ for each group. Intensity values are presented as relative to the internal standard $\beta$-galactosidase and were determined using a luminoanalyzer.

to many organs. In fact, our previous studies have demonstrated increases in lipid hydroperoxides, $\mathrm{F}_{2}$-isoprostan, conjugated diene and proteins, as well as decreases in polyunsaturated lipids, the levels of SNARE proteins, and markedly lowered activities of antioxidant defense systems in the brain nerve terminals of young rats subjected to oxidative stress and normal aged rats $[4,7,8$,
$15,16]$. It has also been found that synaptic vesicles accumulated to abnormal levels in nerve terminals accompanied by a decrease in the release of acetylcholine from nerve terminals $[4,5]$. Furthermore, delayed apoptosis in pyramidal cells and the accumulation of amyloid- $\beta$-like substances in the hippocampus has also been observed in young rats subjected to oxidative stress and normal aged 
rats [17]. In accordance with these phenomena, these rats showed marked deficits in learning and memory functions [17]. Interestingly, these abnormalities are similar to the phenomena observed in AD patients [18]. Thus, it is likely that cognitive impairment may be induced by dysfunctional neurotransmission resulting from neuronal damage caused by oxidative stress.

As previously mentioned, neurotransmission is regulated by the function of synapsin I proteins, which is controlled by CAM kinase II-mediated phosphorylation $[9,10]$. Since oxidative stress-induced alterations in CAM kinase activity and synapsin I levels in nerve terminals remain uncertain, it is necessary to define the influence of oxidative stress on the function of these factors in the neurotransmission process, thereby enhancing understanding of the cause of deficient neurotransmission.

To assess the damage to neurotransmission caused by oxidative stress and aging, we analyzed changes in CAM kinase II activity in the brains of young rats subjected to oxidative stress, vitamin E-deficient young rats and normal aged rats. All groups of animals showed marked increases in the expression of both total CAM kinase II, and CAM kinase II- $\alpha$, which is involved in brain neurotransmission (Figures 1(a) and (b)). These results indicate that oxidative stress and aging induce abnormal expression of CAM kinase II and disrupt brain CAM kinase II activity. It is known that calcium ions and calmodulin are necessary to activate synaptic CAM kinase II. Based on the results obtained in this study, it is likely that oxidative stress increases the levels of calcium ions in nerve terminals, although we did not test this hypothesis. This notion is supported by the finding that free radicals induce damage to nerve terminals, resulting in increased intracellular calcium concentrations [19]. Furthermore, it is known that amyloid- $\beta$, and paired helical filaments (PHF) which is formed by hyperphosphorylated tau proteins, accumulate in the AD brain. It is likely that detection of oxidative stress-induced increases in total CAM kinase II expression, using a CAM kinase II antibody that is partly cross-reactive to a serine (416) residue of tau proteins, may be an indication of the formation of PHF in rats subjected to oxidative stress. Although it is impossible to confirm this hypothesis in the present study, it is interesting to speculate that the existence of PHF accounts for the appearance of amyloid- $\beta$ in the rat brain following oxidative stress, which is likely to occur in Alzheimer's disease.

Phosphorylation of synapsin I is a likely consequence of elevated levels of total CAM kinase II and CAM kinase II- $\alpha$. Whereas the levels of synapsin I markedly decreased following oxidative stress, the levels of phosphorylated synapsin I increased significantly. These results suggest that free synaptic vesicles dissociated from synapsin I and were mobilized toward pre-synaptic mem- branes. Since SNARE proteins, which are involved in docking of synaptic vesicles with pre-synaptic membranes, are damaged by oxidative stress [7], it follows that synaptic vesicles mobilized by oxidative stress do not fuse with pre-synaptic membranes, resulting in abnormal accumulation within the synapse [4,5].

In this study, we assessed the inhibition of these abnormal events by vitamin E. As shown in Figures 3(a) and (b), increases in the expression of CAM kinase II and CAM kinase II- $\alpha$ were markedly inhibited by vitamin E supplementation in either young rats subjected to oxidative stress, or normal aged rats. Furthermore, vita$\min \mathrm{E}$ inhibited the increase in phosphorylated synapsin I and the decrease in total synapsin I (Figures 4(a) and (b)). Thus, it is likely that vitamin $\mathrm{E}$ inhibits these abnormal events through its antioxidant effect. Although the significance of vitamin $\mathrm{E}$ has been proven as a radical chain breaking antioxidant that can protect tissue integrity, vitamin $\mathrm{E}$ has been found to possess functions that are independent of its antioxidative efficacy [20]. In this study, it is impossible to determine the mechanism by which vitamin $\mathrm{E}$ modulates these abnormal events, therefore, further extensive investigation of the inhibitory effect of vitamin $\mathrm{E}$ on oxidative stress and aging are necessary.

\section{CONCLUSION}

The results obtained in this study suggest that rats subjected to oxidative stress exhibit dysfunction in synaptic vesicle-mobilization from the reserve pool. This is reflected in the abnormal activation of CAM kinase II- $\alpha$ and phosphorylation of synapsin I, leading to accumulation of synaptic vesicles in nerve terminals owing to dysfunctional in the membrane fusion process. These results support previous findings where synapsin I was found to be significantly decreased in the stratum radiatum of CA1 in AD patients [21]. Furthermore, it is evident that vitamin $\mathrm{E}$ inhibits the activation of CAM kinase II and the loss of synapsin I caused by oxidative stress, thereby maintaining normal regulation of synaptic vesicle mobilization from the vesicle pool. Considering the previous findings that vitamin $\mathrm{E}$ prevents damage to membrane fusion between synaptic vesicles and pre-synaptic membranes caused by oxidative stress [7,8], it is reasonable that vitamin E may inhibit ROS-induced dysfunction in neurotransmission, leading to improvement in cognitive deficits. The results obtained in this study may, to a certain extent, explain the fact that vitamin E delays institutionalization and the onset of severe dementia in $\mathrm{AD}[22]$.

\section{ACKNOWLEDGEMENTS}

This study has been supported, in part, by MEXT-Supported Program 
for the Strategic Research Foundation at Private University, 2012 and a Grant-in Aid from Eisai Food \& Chemical Co., LTD.

\section{REFERENCES}

[1] Halliwell, B. (1989) Oxidants and the cerebral nervous system: Some fundamental questions. Acta neurologica Scandinavica, 126, 23-33. doi:10.1111/j.1600-0404.1989.tb01779.x

[2] Keller, J.N., Schmitt, F.A., Scheff, S.W., Ding, Q., Chen, Q., Butterfield, D.A. and Markesbery, W.R. (2005) Evidence of increased oxidative damage in subjects with mild cognitive impairment. Neurology, 64, 1152-1156. doi:10.1212/01.WNL.0000156156.13641.BA

[3] Yao, Y., Zhukareva, V., Sung, S., Clark, C.M., Rokach, J., Lee, V.N., Trojanowski, J.Q. and Pratico, D. (2003) Enhanced brain levels of 8,12-iso-iPF2alpha-VI differentiate AD from frontotemporal dementia. Neurology, 26, 436437.

[4] Urano, S., Asai, Y., Makabe, S., Matsu., M, Izumiyama, N., Ohtsubo, K. and Endo, T. (1997) Oxidative injury of synapse and alteration of antioxidative defense systems in rats, and its prevention by vitamin E. European Journal of Biochemistry, 245, 64-70. doi:10.1111/j.1432-1033.1997.00064.X

[5] Urano, S., Sato, Y., Otonari, T., Makabe, S., Suzuki, S., Ogata, M. and Endo, T. (1998) Aging and oxidative stress in neurodegeneration. BioFactor, 7, 103-112. doi:10.1002/biof.5520070114

[6] Fukui, K., Omoi, N., Hayasaka, T., Shinkai, T., Suzuki, S., Abe, K. and Urano, S. (2002) Cognitive impairment of rats caused by oxidative stress and aging, and its prevention by vitamin E. Annals of the New York Academy of Sciences, 959, 275-284.

doi:10.1111/j.1749-6632.2002.tb02099.x

[7] Kaneai, N., Arai, M., Takatsu, H., Fuki, K. and Urano, S. (2012) Vitamin E inhibits oxidative stress-induced denaturation of nerve terminal proteins involved in neurotransmission. Journal of Alzheimer's Disease, 28, 183189.

[8] Arai, M., Saitoh, M., Takaysu, H., Fukui, K. and Urano, S. (2011) Dysfunction of the fusion of pre-synaptic plasma membranes and synaptic vesicles caused by oxidative stress, and its prevention by vitamin E. Journal of Alzheimer's Disease, 24, 759-766.

[9] Hilfiker, S., Pierobone, V.A., Czernik, A.J., Kao, H.-T., Augustine, G.J. and Greengard, P. (1999) Synapsins as regulators of neurotransmitter release. Philosophical Transactions of the Royal Society of London. Series B, Biological Sciences, 354, 269-279. doi:10.1098/rstb.1999.0378

[10] Greengard, P., Valtorta, F., Czernik, A.J. and Benfenati, F. (1993) Synaptic vesicle phosphoproteins and regulation of synaptic function. Science, 259, 780-785. doi:10.1126/science. 8430330

[11] Fukui, K. and Urano, S. (2007) Neuronal damage, cogni- tive impairment and alpha-tocopherol. In: Preedy, V.R. and Watson, R.R., Eds., The Encyclopedia of Vitamin E, CAB International, Oxon, 454-460.

[12] Dodd, P.R., Hardy, J.R., Oakley, A.E., Edwardson, J.A., Perry, E.K. and Delaunoy, J.P. (1981) A rapid method for preparing synaptosomes: Comparison with alternative procedures. Brain Research, 226, 107-118. doi:10.1016/0006-8993(81)91086-6

[13] Harman, D. (1956) Aging: A theory based on free radical and radiation chemistry. Journal of Gerontology, 11, 298300. doi:10.1093/geronj/11.3.298

[14] Harman, D. (1984) Free radical theory of aging: The free radical diseases. Age, 7, 111-131. doi:10.1007/BF02431866

[15] Onodera, K., Omoi, N., Fuki, K., Hayasaka, T., Shinkai, T., Suzuki, S., Abe, K. and Urano, S. (2003) Oxidative damage of rat cerebral cortex and hippocampus, and changes in antioxidative defense systems caused by hyperoxia. Free Radical Research, 37, 367-372. doi:10.1080/1071576031000090019

[16] Nishio, T., Miyadera, R., Sakai, R., Abe, K., Kanazawa, H., Fukui, K. and Urano, S. (2006) Increased $\mathrm{F}_{2}$-isoprostane levels in the rat brain and plasma caused by oxidative stress and aging, and inhibitory effect of vitamin E. Journal Clinical Biochemistry and Nutrition, 38, 161-166. doi:10.3164/jcbn.38.161

[17] Fukui, K., Takatsu, H., Shinkai, T., Suzuki, S., Abe, K. and Urano, S. (2005) Appearance of amyloid- $\beta$-like substances and delayed-type apoptosis in rat hippocampus CA1 region through aging and oxidative stress. Journal of Alzheimer's Disease, 8, 299-309.

[18] Zhu, X., Smith, M.A., Honda, K., Aliev, G., Moreira, P.I., Nunomura, A., Casadesus, G., Harris, P.L., Siedlak, S.L. and Perry, G. (2007) Vascular oxidative stress in Alzheimer's disease. Journal of the Neurological Sciences, 257, 240-246. doi:10.1016/j.jns.2007.01.039

[19] Tretter, L. and Adam-Vizi, V. (1996) Early events in free radical-mediated damage of isolated nerve terminals: Effect of peroxides on membrane potential and intracellular $\mathrm{Na}^{+}$and $\mathrm{Ca}^{2+}$ concentrations. Journal of Neurochemistry, 66, 2057-2066. doi:10.1046/j.1471-4159.1996.66052057.x

[20] Azzi, A. and Stocker, A. (2000) Vitamin E: Non-antioxidant roles. Progress in Lipid Research, 39, 231-255. doi:10.1016/S0163-7827(00)00006-0

[21] Qin, S., Hu, X.Y., Xu, H. and Zhou, J.N. (2004) Regional alteration of synapsin I in the hippocampal formation of Alzheimer's disease patients. Acta Neuropathologica, 107, 209-215. doi:10.1007/s00401-003-0800-4

[22] Sano, M., Ernest, C., Thomas, R.G., Klauber, M.R., Schafer, K., Grundman, M., Woodbury, P., Cotman, C.W., Pfeiffe, R.E., Schneide, R.L.S. and Thal, L.J. (1997) A controlled trial of selegiline, alpha-tocopherol, or both as treatment for Alzheimer's disease. The Alzheimer's disease cooperative study. The New England Journal of Medicine, 336, 1216-1222. doi:10.1056/NEJM199704243361704 